\title{
Screening of Different Chilli Genotypes against Anthracnose Disease (Colletotrichum capsici) under Controlled Condition
}

\author{
Veerendra Gupta ${ }^{1 *}$, Arvinder Kaur ${ }^{1}$, Akanksha Singh ${ }^{2}$, \\ Himanshu Shekhar ${ }^{3}$, Reeti Singh ${ }^{1}$ and Ashish Bobde ${ }^{1}$
}

\author{
${ }^{1}$ Department of Plant Pathology, College of Agriculture, RVSKVV, Gwalior \\ (M.P.)-474002, India \\ ${ }^{2}$ Department of Plant Pathology, GBPUA\&T, Pantnagar, Uttrakhand, India \\ ${ }^{3}$ Department of Plant Breeding BCKV West Bengal, India
}

*Corresponding author

\section{A B S T R A C T}

\section{Keywords}

Cultivars,

Germplasms, PDI,

Disease intensity percent

\section{Article Info}

Accepted:

20 February 2018

Available Online:

10 March 2018
Chilli (Capsicum апnиum) is an important spice as well as vegetable crop that are grown throughout the world especially in tropical and subtropical regions. India is a major producer, exporter and consumer of chilli. Chilli is grown in almost all states. Anthracnose disease can occur on leaves, stems, and both pre and post-harvest fruits. It is one of the major and devastating diseases of chilli causes severe losses $(10-60 \%)$ both in yield and quality of the chilli depending upon the varieties. Diseases are the major problem for successful cultivation of chilli in India. For successful cultivation of chilli, it is important to identify resistant and tolerant varieties against anthracnose diseases. Therefore, experiment was conducted in the Department of Plant Pathology, college of Agriculture Gwalior and the field experiment were carried out in the Krishi Vigyan Kendra Research farm, RVSKVV Gwalior (M.P.) using CRD with 3 replication during kharif 2015-16. For Screening of 25 varieties \& genotypes of chilli received from ICAR/National institutes were evaluated against anthracnose (Colletotricum capsici) under laboratory condition using three artificial inoculation treatment viz distilled water inoculation, without injury inoculation, injury + inoculation. Under laboratory/controlled condition, Semiripe full mature chilli fruits ( 25 days old) were chosen for this study. Fruits were carefully detached from plants and washed with sterile distilled water (SDW). Therefore suspension of conidia $\left(5 \times 10^{5}\right.$ conidia $\mathrm{ml}^{-1}$ water) of pathogen isolate was prepared and drops of conidial suspension were placed at proximal and distal ends on each fruit after puncturing the fruit $\left(0.6 \mathrm{~mm}\right.$ diameter $\times 1.2 \mathrm{~mm}$ depth). Three sets of fruits were prepared for experiment. In $1^{\text {st }}$ set, two

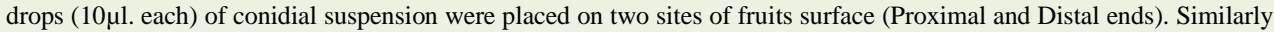

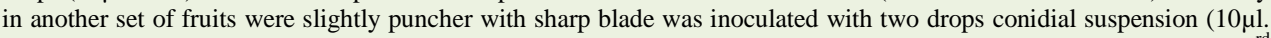
Each) on two sites (Proximal and Distal ends) of fruits observed anthracnose lesion development on fruit surface. In $3^{\text {rd }}$ sets of fruits, sterilized distilled water was used instead of conidial suspension, which acts as control. After inoculation, the chilli fruits were placed in a plastic container/ box $\left(30 \times 20 \times 7 \mathrm{~cm}^{3}\right)$ lined with four layers of paper towel moistened with sterile distilled water to produce a well humid environment and later sealed with a plastic tape. Symptoms on the chilli fruit were examined and evaluated after 5, 7, and 10 days after inoculation by measuring the area of the lesion for disease development or \% disease intensity. The PDI was observed on infected fruits. The percent disease severity on fruits was recorded by using 0-5 scale. The disease reaction of each genotype was categorised on the basis of following rating scale viz., $0 \%$ - immune, 0 to $5 \%$ - resistant, 5 to $25 \%$ - moderately resistant, 25 to $50 \%$ - susceptible, above $50 \%$ highly susceptible. The genotypes were rated as resistant and susceptible based on the range of lesion area $(\mathrm{mm})$ or of the disease incidence (\%). Among the artificial inoculation, two drops of conidial suspension after injury the fruit was found to be best treatment of inoculation and no infection noticed in other remaining both treatments. It was observed that injury to the fruits favoured the C. capsici development on detached fruit surface. Varieties Arka Harita, Classica-152 and Madhurima-148 were showing resistance reaction under two drops of conidial suspension after injury the fruits while EC-341075, Pusa Jwala, Pant C-1, Arka Meghna, LAC-434 and Sonakshi-44 were show moderately resistance reaction as compared to rest of varieties after five, seven and ten days of inoculation. 


\section{Introduction}

Chilli (Capsicum annuum) is an important spice as well as vegetable crop cultivated worldwide. In India, the major chillies growing states are Andhra Pradesh, Karnataka, Maharashtra, Orissa, Tamil Nadu, Madhya Pradesh and Rajasthan. In Madhya Pradesh chilli is grown under an area of 54.41thousand ha with production of 93.57 thousand MT and productivity 0.98 ton per ha (Anon-2013-14).

Colletotrichum spp. are among the most important plant pathogen worldwide, causing the economically important disease anthracnose (die back or fruit rot, leaf spot, wilt, damping off, etc) in a wide range of hosts, including cereals, legumes, vegetables and tree fruits (Bailey and Jeger, 1992).

Anthracnose of chilli (Capsicum annuum L.) caused by Colletotrichum capsici (Syd.) Butler and Bisby, is one of the major and devastating diseases of chilli causes severe losses (10-60\%) both in yield and quality of the chilli depending upon the varieties (Bansal and Grover, 1969). Management strategies for this disease include use of disease free seed, resistant/tolerant cultivars and fungicidal sprays. The present investigation was aimed to identify the resistant cultivars/germplasms against Colletotrichum capsici under field condition by artificial inoculation. Where the 25 germplasms were screened against Colletotrichum capsici in search of resistant cultivars/germplasms.

\section{Materials and Methods}

The experiment was conducted in the Department of Plant Pathology, college of Agriculture Gwalior and the field experiment were carried out in the Krishi Vigyan Kendra Research farm, RVSKVV Gwalior (M.P.) using Completely Randomised Block Design
(CRD) with three replication during Kharif 2015-16. Twenty five cultivars (Kashi Anmol, Kashi Gaurav, Pant C-1, Punjab Lal, Pusa Jwala, Pusa Sadabahar, AKC 89-38, Jayanti, EC-566320, EC-341075, P-1649, LAC-434, LAC-422, Phule Jyoti, Arka Khyati, Arka Meghna, Arka Lohit, Arka Harita, Jawahar Mirch-218, Jawahar Mirch-283, Classica-152, Divya Jyoti, Madhurima -148, Natasha -727 , Sonakshi-44) or Germplasms of chilli were screened against anthracnose (Colletotricum capsici) under laboratory condition using three artificial inoculation treatment under laboratory condition viz., distilled water inoculation, without injury inoculation, injury + inoculation.

\section{Methodology}

\section{Screening of chilli cultivars}

Semi ripe chilli fruits (25 days old) of 25 varieties/ germplasm were chosen for this study. A purified and pathogenic isolated fungus (Colletotrichum capsici) was used as a screening agent based on its pathogenicity. The study was conducted using three artificial innoculation treatment under laboratory condition viz distilled water inoculation, without injury inoculation, injury + inoculation.

\section{Preparation of spore suspension and inoculation}

Conidia of the fungus were collected from actively growing ten days old culture. The spores were harvested by adding sterilized distilled water into petri plates and scraped gently with camel hair brush.

Water loaded with spore mass in petriplates was collected into a beaker and filtered. The concentration of spore suspension was adjusted to $10^{6}$ spores $/ \mathrm{ml}$. by observing under microscope. 
Artificial inoculations by fruit puncture method under laboratory conditions

Sets of chilli fruits belonging to, dark green mature $(2-3 \mathrm{~cm}$ long fruits, fully grown fruits before red color initiation) were obtained from healthy chilli plants. Semi ripe chilli fruits $(25$ days old) of 25 varieties/ germplasm were chosen for this study. Fruits were carefully detached from plants and washed with sterile distilled water (SDW) and then wiped with cotton wools soaked in ethanol to reduce microbes on the surface. It has been reported that suitable concentration of conidia in inoculums drop is $5 \times 10^{5}$ conidia per $\mathrm{ml}$ of water (Rajapakse, 1998). Therefore suspension of conidia $\left(5 \times 10^{5}\right.$ conidia $\mathrm{ml}^{-1}$ water) of pathogen isolate was prepared and drops of conidial suspension was placed at proximal and distal ends on each fruit after puncturing the fruit $(0.6 \mathrm{~mm}$ diameter $\times 1.2$ mm depth)

Three sets of fruits were prepared for experiment. In $1^{\text {st }}$ set, two drops $(10 \mu 1$. each) of conidial suspension were placed on two sites of fruits surface (Proximal and Distal ends). Similarly in another set of fruits were slightly puncher with sharp blade was inoculated with two drops conidial suspension (10 $\mu 1$. Each) on two sites (Proximal and Distal ends) of fruits to observed anthracnose lesion development on fruit surface, in $3^{\text {rd }}$ sets of fruits, only sterilized distilled water was used instead of conidial suspension, which act as control. After inoculation, the chilli fruits were placed in a plastic container/box $(30 \times 20 \times 7$ $\mathrm{cm}^{3}$ ) lined with four layers of paper towel moistened with sterile distilled water to produce a humid environment and later sealed with a plastic tape. Symptoms on the chilli fruit were examined and evaluated after 5, 7, and 10 days by measuring the area of the lesion for disease development. The experiment involved a completely randomized design with three replicates.
The PDI was observed on infected leaves/Fruits. The percent disease severity on leaf or fruits was recorded by using 0-5 scale (Jeyalakshmi and Seetharaman, 1998) where 0- no disease, 1- up to 5\%, 2- 5 to $10 \%, 3-10$ to $25 \%, 4-25$ to $50 \%$ and 5 - above $50 \%$. The percent disease index was calculated by using following formula.

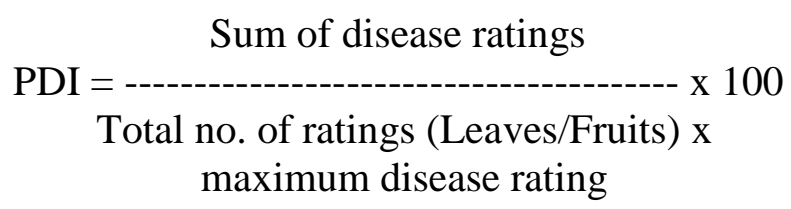

The disease reaction of each genotype was categorised on the basis of following rating scale (Singh et al., 1993) viz., 0\%- immune, 0 to $5 \%$ - resistant, 5 to $25 \%$ - moderately resistant, 25 to $50 \%$ - susceptible, above 50\% highly susceptible and Data was analysed by ANOVA through completely randomised blocked design.

The genotypes were rated as resistant and susceptible based on the range of lesion area $(\mathrm{mm})$ or of the disease incidence $(\%)$.

\section{Results and Discussion}

The reaction of isolated $C$. capsici against a set of twenty five varieties of chilli was assessed in controlled conditions. Two drops of sterile distilled water without injury the fruit, drop of conidial suspension after without injury the fruit and drop of conidial suspension after injury the fruits were tested under in vitro condition and data were recorded from five, seven and ten days after inoculation. It is evident from table 1, that significant differences were noticed in all varieties with respect to artificial screening under laboratory condition. Among the artificial inoculation, two drops of conidial suspension after injury the fruit was found to be best method of inoculation and no infection noticed in other remaining both treatments. 
Table.1 Reaction of chilli varieties/germplasms against anthracnose under artificial inoculation in laboratory condition after 5,7 and 10 days of inoculation

\begin{tabular}{|c|c|c|c|c|c|c|c|c|c|c|c|c|}
\hline \multirow[t]{3}{*}{ Varieties } & \multicolumn{12}{|c|}{ Per cent Disease Intensity* } \\
\hline & \multicolumn{4}{|c|}{ Five days after inoculation } & \multicolumn{4}{|c|}{ Seven days after inoculation } & \multicolumn{4}{|c|}{ Ten days after inoculation } \\
\hline & $\begin{array}{c}\text { Distilled } \\
\text { water } \\
\text { inoculatio } \\
n\end{array}$ & $\begin{array}{c}\text { Without } \\
\text { injury } \\
\text { inoculation }\end{array}$ & $\begin{array}{c}\text { Injury } \\
\text { inoculatio } \\
\mathbf{n}\end{array}$ & $\begin{array}{c}\text { Mean } \\
\text { A }\end{array}$ & $\begin{array}{c}\text { Distilled } \\
\text { water } \\
\text { inoculatio } \\
n\end{array}$ & $\begin{array}{c}\text { Without } \\
\text { injury } \\
\text { inoculation }\end{array}$ & $\begin{array}{c}\text { Injury } \\
\text { inoculatio } \\
n\end{array}$ & Mean A & $\begin{array}{c}\text { Distilled } \\
\text { water } \\
\text { inoculatio } \\
n\end{array}$ & $\begin{array}{c}\text { Without } \\
\text { injury } \\
\text { inoculation }\end{array}$ & $\begin{array}{c}\text { Injury } \\
\text { inoculatio } \\
\mathbf{n}\end{array}$ & Mean A \\
\hline $\begin{array}{c}\text { Kashi } \\
\text { Anmol }\end{array}$ & $0.00(0.00)$ & $0.00(0.00)$ & $\begin{array}{c}36.29 \\
(35.05)\end{array}$ & $\begin{array}{c}11.68 \\
(12.10)\end{array}$ & $0.00(0.00)$ & $0.00(0.00)$ & $\begin{array}{c}36.50 \\
(37.15)\end{array}$ & $\begin{array}{c}12.17 \\
(12.38)\end{array}$ & $0.00(0.00)$ & $0.00(0.00)$ & $\begin{array}{c}41.00 \\
(39.80)\end{array}$ & $\begin{array}{c}13.67 \\
(13.27)\end{array}$ \\
\hline $\begin{array}{c}\text { Kashi } \\
\text { Gaurav }\end{array}$ & $0.00(0.00)$ & $0.00(0.00)$ & $\begin{array}{c}43.26 \\
(47.00)\end{array}$ & $\begin{array}{c}15.67 \\
(14.42)\end{array}$ & $0.00(0.00)$ & $0.00(0.00)$ & $\begin{array}{c}49.50 \\
(44.70)\end{array}$ & $\begin{array}{c}16.50 \\
(14.90)\end{array}$ & $0.00(0.00)$ & $0.00(0.00)$ & $\begin{array}{c}57.50 \\
(49.30)\end{array}$ & $\begin{array}{c}19.17 \\
(16.43)\end{array}$ \\
\hline Pant C-1 & $0.00(0.00)$ & $0.00(0.00)$ & $\begin{array}{l}17.31 \\
(9.01)\end{array}$ & $\begin{array}{c}5.77 \\
(3.00)\end{array}$ & $0.00(0.00)$ & $0.00(0.00)$ & $\begin{array}{c}21.50 \\
(27.84)\end{array}$ & $7.17(9.28)$ & $0.00(0.00)$ & $0.00(0.00)$ & $\begin{array}{c}23.00 \\
(27.40)\end{array}$ & $\begin{array}{c}7.67(9.1 \\
3)\end{array}$ \\
\hline $\begin{array}{c}\text { Punjab } \\
\text { Lal }\end{array}$ & $0.00(0.00)$ & $0.00(0.00)$ & $\begin{array}{c}52.81 \\
(63.50)\end{array}$ & $\begin{array}{c}21.17 \\
(17.60)\end{array}$ & $0.00(0.00)$ & $0.00(0.00)$ & $\begin{array}{c}65.50 \\
(54.01)\end{array}$ & $\begin{array}{c}21.83 \\
(18.00)\end{array}$ & $0.00(0.00)$ & $0.00(0.00)$ & $\begin{array}{c}71.00 \\
(57.40)\end{array}$ & $\begin{array}{c}23.67 \\
(19.13)\end{array}$ \\
\hline $\begin{array}{l}\text { Pusa } \\
\text { Jwala }\end{array}$ & $0.00(0.00)$ & $0.00(0.00)$ & $\begin{array}{l}17.25 \\
(8.80)\end{array}$ & $\begin{array}{c}2.93 \\
(5.75)\end{array}$ & $0.00(0.00)$ & $0.00(0.00)$ & $\begin{array}{l}14.96 \\
(22.74)\end{array}$ & $\begin{array}{c}4.99 \\
(7.58)\end{array}$ & $0.00(0.00)$ & $0.00(0.00)$ & $\begin{array}{c}20.68 \\
(27.04)\end{array}$ & $\begin{array}{c}6.89 \\
(9.01)\end{array}$ \\
\hline $\begin{array}{c}\text { Pusa } \\
\text { Sadabahar }\end{array}$ & $0.00(0.00)$ & $0.00(0.00)$ & $\begin{array}{c}46.70 \\
(53.00)\end{array}$ & $\begin{array}{c}17.67 \\
(15.57)\end{array}$ & $0.00(0.00)$ & $0.00(0.00)$ & $\begin{array}{c}55.00 \\
(47.85)\end{array}$ & $\begin{array}{c}18.33 \\
(15.95)\end{array}$ & $0.00(0.00)$ & $0.00(0.00)$ & $\begin{array}{c}59.00 \\
(50.17)\end{array}$ & $\begin{array}{c}19.67 \\
(16.72)\end{array}$ \\
\hline $\begin{array}{c}\text { AKC 89- } \\
38\end{array}$ & $0.00(0.00)$ & $0.00(0.00)$ & $\begin{array}{c}35.05 \\
(33.00)\end{array}$ & $\begin{array}{c}11.00 \\
(11.68)\end{array}$ & $0.00(0.00)$ & $0.00(0.00)$ & $\begin{array}{c}35.00 \\
(36.26)\end{array}$ & $\begin{array}{c}11.67 \\
(12.09)\end{array}$ & $0.00(0.00)$ & $0.00(0.00)$ & $\begin{array}{c}40.50 \\
(39.51)\end{array}$ & $\begin{array}{c}13.50 \\
(13.17)\end{array}$ \\
\hline Jayanti & $0.00(0.00)$ & $0.00(0.00)$ & $\begin{array}{c}50.17 \\
(59.00)\end{array}$ & $\begin{array}{c}19.67 \\
(16.72)\end{array}$ & $0.00(0.00)$ & $0.00(0.00)$ & $\begin{array}{c}63.00 \\
(52.52)\end{array}$ & $\begin{array}{c}21.00 \\
(17.51)\end{array}$ & $0.00(0.00)$ & $0.00(0.00)$ & $\begin{array}{c}65.50 \\
(54.01)\end{array}$ & $\begin{array}{c}21.83 \\
(18.00)\end{array}$ \\
\hline $\begin{array}{c}\text { EC- } \\
566320\end{array}$ & $0.00(0.00)$ & $0.00(0.00)$ & $\begin{array}{c}42.69 \\
(46.00)\end{array}$ & $\begin{array}{c}15.33 \\
(14.23)\end{array}$ & $0.00(0.00)$ & $0.00(0.00)$ & $\begin{array}{c}48.00 \\
(43.84)\end{array}$ & $\begin{array}{c}16.00 \\
(14.61)\end{array}$ & $0.00(0.00)$ & $0.00(0.00)$ & $\begin{array}{c}51.00 \\
(45.56)\end{array}$ & $\begin{array}{c}17.00 \\
(15.19)\end{array}$ \\
\hline $\begin{array}{c}\text { EC- } \\
341075\end{array}$ & $0.00(0.00)$ & $0.00(0.00)$ & $\begin{array}{l}16.18 \\
(7.78)\end{array}$ & $\begin{array}{c}2.59 \\
(5.40)\end{array}$ & $0.00(0.00)$ & $0.00(0.00)$ & $\begin{array}{c}13.23 \\
(21.31)\end{array}$ & $\begin{array}{c}4.41 \\
(7.11)\end{array}$ & $0.00(0.00)$ & $0.00(0.00)$ & $\begin{array}{c}18.28 \\
(25.30)\end{array}$ & $\begin{array}{c}6.09 \\
(8.43)\end{array}$ \\
\hline P-1649 & $0.00(0.00)$ & $0.00(0.00)$ & $\begin{array}{c}55.53 \\
(68.00)\end{array}$ & $\begin{array}{c}22.67 \\
(18.51)\end{array}$ & $0.00(0.00)$ & $0.00(0.00)$ & $\begin{array}{c}73.00 \\
(58.67)\end{array}$ & $\begin{array}{c}24.33 \\
(19.56)\end{array}$ & $0.00(0.00)$ & $0.00(0.00)$ & $\begin{array}{c}77.50 \\
(61.66)\end{array}$ & $\begin{array}{c}25.83 \\
(20.55)\end{array}$ \\
\hline LAC-434 & $0.00(0.00)$ & $0.00(0.00)$ & $\begin{array}{c}21.22 \\
(13.13)\end{array}$ & $\begin{array}{c}4.38 \\
(7.07)\end{array}$ & $0.00(0.00)$ & $0.00(0.00)$ & $\begin{array}{c}22.31 \\
(28.16)\end{array}$ & $\begin{array}{c}7.44 \\
(9.39)\end{array}$ & $0.00(0.00)$ & $0.00(0.00)$ & $\begin{array}{l}24.03 \\
(29.34)\end{array}$ & $\begin{array}{c}8.01 \\
(9.78)\end{array}$ \\
\hline LAC-422 & $0.00(0.00)$ & $0.00(0.00)$ & $\begin{array}{c}48.43 \\
(56.00)\end{array}$ & $\begin{array}{c}18.67 \\
(16.14)\end{array}$ & $0.00(0.00)$ & $0.00(0.00)$ & $\begin{array}{c}59.00 \\
(50.17)\end{array}$ & $\begin{array}{c}19.67 \\
(16.72)\end{array}$ & $0.00(0.00)$ & $0.00(0.00)$ & $\begin{array}{c}63.00 \\
(52.52)\end{array}$ & $\begin{array}{c}21.00 \\
(17.51)\end{array}$ \\
\hline $\begin{array}{l}\text { Phule } \\
\text { Jyoti }\end{array}$ & $0.00(0.00)$ & $0.00(0.00)$ & $\begin{array}{c}51.34 \\
(61.00)\end{array}$ & $\begin{array}{c}20.33 \\
(17.11)\end{array}$ & $0.00(0.00)$ & $0.00(0.00)$ & $\begin{array}{l}63.50 \\
(52.81)\end{array}$ & $\begin{array}{c}21.17 \\
(17.60)\end{array}$ & $0.00(0.00)$ & $0.00(0.00)$ & $\begin{array}{l}69.00 \\
(56.15)\end{array}$ & $\begin{array}{c}23.00 \\
(18.72)\end{array}$ \\
\hline $\begin{array}{c}\text { Arka } \\
\text { Khyati }\end{array}$ & $0.00(0.00)$ & $0.00(0.00)$ & $\begin{array}{c}55.22 \\
(67.50)\end{array}$ & $\begin{array}{c}22.50 \\
(18.41)\end{array}$ & $0.00(0.00)$ & $0.00(0.00)$ & $\begin{array}{l}69.00 \\
(56.14)\end{array}$ & $\begin{array}{c}23.00 \\
(18.72)\end{array}$ & $0.00(0.00)$ & $0.00(0.00)$ & $\begin{array}{c}74.00 \\
(59.32)\end{array}$ & $\begin{array}{c}24.67 \\
(19.77)\end{array}$ \\
\hline $\begin{array}{c}\text { Arka } \\
\text { Meghna }\end{array}$ & $0.00(0.00)$ & $0.00(0.00)$ & $\begin{array}{c}19.71 \\
(11.43)\end{array}$ & $\begin{array}{c}3.81 \\
(6.57)\end{array}$ & $0.00(0.00)$ & $0.00(0.00)$ & $\begin{array}{c}19.42 \\
(26.09)\end{array}$ & $\begin{array}{c}6.47 \\
(8.70)\end{array}$ & $0.00(0.00)$ & $0.00(0.00)$ & $\begin{array}{c}23.51 \\
(28.99)\end{array}$ & $\begin{array}{c}7.84 \\
(9.66)\end{array}$ \\
\hline $\begin{array}{l}\text { Arka } \\
\text { Lohit }\end{array}$ & $0.00(0.00)$ & $0.00(0.00)$ & $\begin{array}{c}40.67 \\
(42.50)\end{array}$ & $\begin{array}{c}14.17 \\
(13.56)\end{array}$ & $0.00(0.00)$ & $0.00(0.00)$ & $\begin{array}{c}45.50 \\
(42.40)\end{array}$ & $\begin{array}{c}15.17 \\
(14.13)\end{array}$ & $0.00(0.00)$ & $0.00(0.00)$ & $\begin{array}{c}51.50 \\
(45.84)\end{array}$ & $\begin{array}{c}17.17 \\
(15.28)\end{array}$ \\
\hline $\begin{array}{c}\text { Arka } \\
\text { Harita }\end{array}$ & $0.00(0.00)$ & $0.00(0.00)$ & $9.39(2.68)$ & $\begin{array}{c}0.89 \\
(3.13)\end{array}$ & $0.00(0.00)$ & $0.00(0.00)$ & $\begin{array}{c}3.61 \\
(10.92)\end{array}$ & $\begin{array}{c}1.20 \\
(3.64)\end{array}$ & $0.00(0.00)$ & $0.00(0.00)$ & $\begin{array}{c}3.93 \\
(11.40)\end{array}$ & $\begin{array}{c}1.31 \\
(3.80)\end{array}$ \\
\hline $\begin{array}{c}\text { Jawahar } \\
\text { Mirch-218 }\end{array}$ & $0.00(0.00)$ & $0.00(0.00)$ & $\begin{array}{c}43.26 \\
(47.00)\end{array}$ & $\begin{array}{c}15.67 \\
(14.42)\end{array}$ & $0.00(0.00)$ & $0.00(0.00)$ & $\begin{array}{c}49.00 \\
(44.41)\end{array}$ & $\begin{array}{c}16.33 \\
(14.80)\end{array}$ & $0.00(0.00)$ & $0.00(0.00)$ & $\begin{array}{c}54.50 \\
(47.56)\end{array}$ & $\begin{array}{c}18.17 \\
(15.85)\end{array}$ \\
\hline $\begin{array}{c}\text { Jawahar } \\
\text { Mirch-283 }\end{array}$ & $0.00(0.00)$ & $0.00(0.00)$ & $\begin{array}{l}56.46 \\
(69.50)\end{array}$ & $\begin{array}{c}23.17 \\
(18.82)\end{array}$ & $0.00(0.00)$ & $0.00(0.00)$ & $\begin{array}{c}72.50 \\
(58.35)\end{array}$ & $\begin{array}{c}24.17 \\
(19.45)\end{array}$ & $0.00(0.00)$ & $0.00(0.00)$ & $\begin{array}{c}77.50 \\
(61.66)\end{array}$ & $\begin{array}{c}25.83 \\
(20.55)\end{array}$ \\
\hline $\begin{array}{c}\text { Classica- } \\
152\end{array}$ & $0.00(0.00)$ & $0.00(0.00)$ & $9.48(2.78)$ & $\begin{array}{c}0.93 \\
(3.16)\end{array}$ & $0.00(0.00)$ & $0.00(0.00)$ & $\begin{array}{c}3.75 \\
(11.03)\end{array}$ & $\begin{array}{c}1.25 \\
(3.68)\end{array}$ & $0.00(0.00)$ & $0.00(0.00)$ & $\begin{array}{c}4.51 \\
(12.24)\end{array}$ & $\begin{array}{c}1.50 \\
(4.08)\end{array}$ \\
\hline $\begin{array}{l}\text { Divya } \\
\text { Jyoti }\end{array}$ & $0.00(0.00)$ & $0.00(0.00)$ & $\begin{array}{c}35.35 \\
(33.50)\end{array}$ & $\begin{array}{c}11.17 \\
(11.78)\end{array}$ & $0.00(0.00)$ & $0.00(0.00)$ & $\begin{array}{c}37.50 \\
(37.75)\end{array}$ & $\begin{array}{c}12.50 \\
(12.58)\end{array}$ & $0.00(0.00)$ & $0.00(0.00)$ & $\begin{array}{c}41.00 \\
(39.80)\end{array}$ & $\begin{array}{c}13.67 \\
(13.27)\end{array}$ \\
\hline $\begin{array}{c}\text { Madhurim } \\
\text { a -148 }\end{array}$ & $0.00(0.00)$ & $0.00(0.00)$ & $8.95(2.43)$ & $\begin{array}{c}0.81 \\
(2.98)\end{array}$ & $0.00(0.00)$ & $0.00(0.00)$ & $\begin{array}{c}3.27 \\
(10.40)\end{array}$ & $\begin{array}{c}1.09 \\
(3.47)\end{array}$ & $0.00(0.00)$ & $0.00(0.00)$ & $\begin{array}{c}4.39 \\
(12.08)\end{array}$ & $\begin{array}{c}1.46 \\
(4.03)\end{array}$ \\
\hline $\begin{array}{c}\text { Natasha - } \\
727\end{array}$ & $0.00(0.00)$ & $0.00(0.00)$ & $\begin{array}{c}50.17 \\
(59.00)\end{array}$ & $\begin{array}{c}19.67 \\
(16.72)\end{array}$ & $0.00(0.00)$ & $0.00(0.00)$ & $\begin{array}{c}63.00 \\
(52.52)\end{array}$ & $\begin{array}{c}7.66 \\
(17.51)\end{array}$ & $0.00(0.00)$ & $0.00(0.00)$ & $\begin{array}{c}66.00 \\
(54.32)\end{array}$ & $\begin{array}{c}22.00 \\
(18.11)\end{array}$ \\
\hline $\begin{array}{c}\text { Sonakshi- } \\
44\end{array}$ & $0.00(0.00)$ & $0.00(0.00)$ & $\begin{array}{c}21.56 \\
(13.53)\end{array}$ & $\begin{array}{c}4.51 \\
(7.19)\end{array}$ & $0.00(0.00)$ & $0.00(0.00)$ & $\begin{array}{c}22.99 \\
(28.63)\end{array}$ & $\begin{array}{l}21.00 \\
(9.54)\end{array}$ & $0.00(0.00)$ & $0.00(0.00)$ & $\begin{array}{c}21.37 \\
(27.51)\end{array}$ & $\begin{array}{c}7.12 \\
(9.17)\end{array}$ \\
\hline Mean B & $0.00(0.00)$ & $0.00(0.00)$ & $\begin{array}{c}36.86 \\
(38.76)\end{array}$ & & $0.00(0.00)$ & $0.00(0.00)$ & $\begin{array}{c}42.26 \\
(39.39)\end{array}$ & & $0.00(0.00)$ & $0.00(0.00)$ & $\begin{array}{c}46.05 \\
(41.83)\end{array}$ & \\
\hline Factors & SE(m) & C.D. & \multicolumn{2}{|c|}{ C.V. } & \multicolumn{2}{|c|}{ SE(m) } & C.D. & C.V. & \multicolumn{2}{|c|}{ SE(m) } & C.D. & C.V. \\
\hline Factor(A) & 0.15 & 0.41 & \multicolumn{2}{|c|}{9.79} & \multicolumn{2}{|c|}{0.18} & 0.51 & 10.53 & \multicolumn{2}{|c|}{0.15} & 0.41 & 9.37 \\
\hline Factor(B) & 0.05 & 0.14 & \multicolumn{2}{|c|}{5.65} & \multicolumn{2}{|c|}{0.06} & 0.18 & 6.08 & \multicolumn{2}{|c|}{0.05} & 0.14 & 5.41 \\
\hline $\begin{array}{c}\text { Factor(A } \\
\text { X B) }\end{array}$ & 0.26 & 0.72 & \multicolumn{2}{|c|}{12.89} & \multicolumn{2}{|c|}{0.32} & 0.89 & 14.05 & \multicolumn{2}{|c|}{0.25} & 0.72 & 12.10 \\
\hline
\end{tabular}


Effect of different methods of inoculation with C.Capsici after 10 days of inoculation

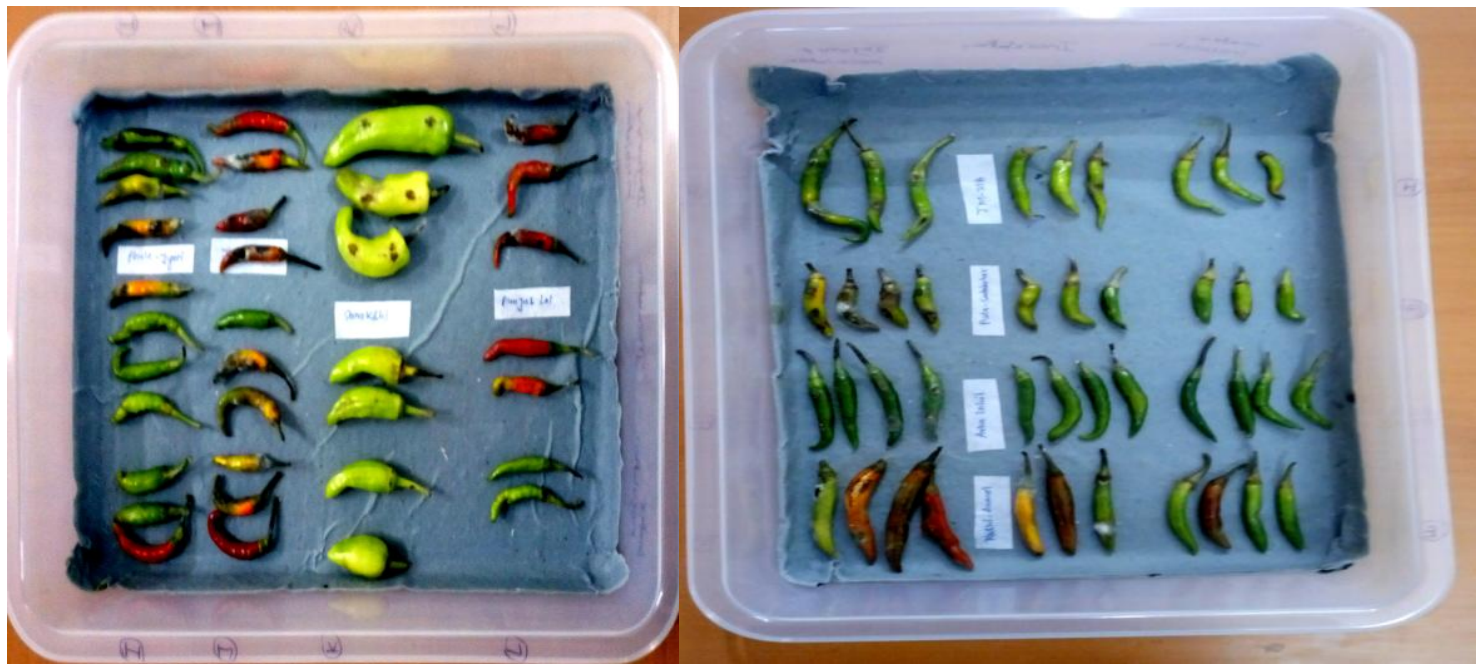

Effect of different methods of inoculation with C.Capsici after 10 days of inoculation

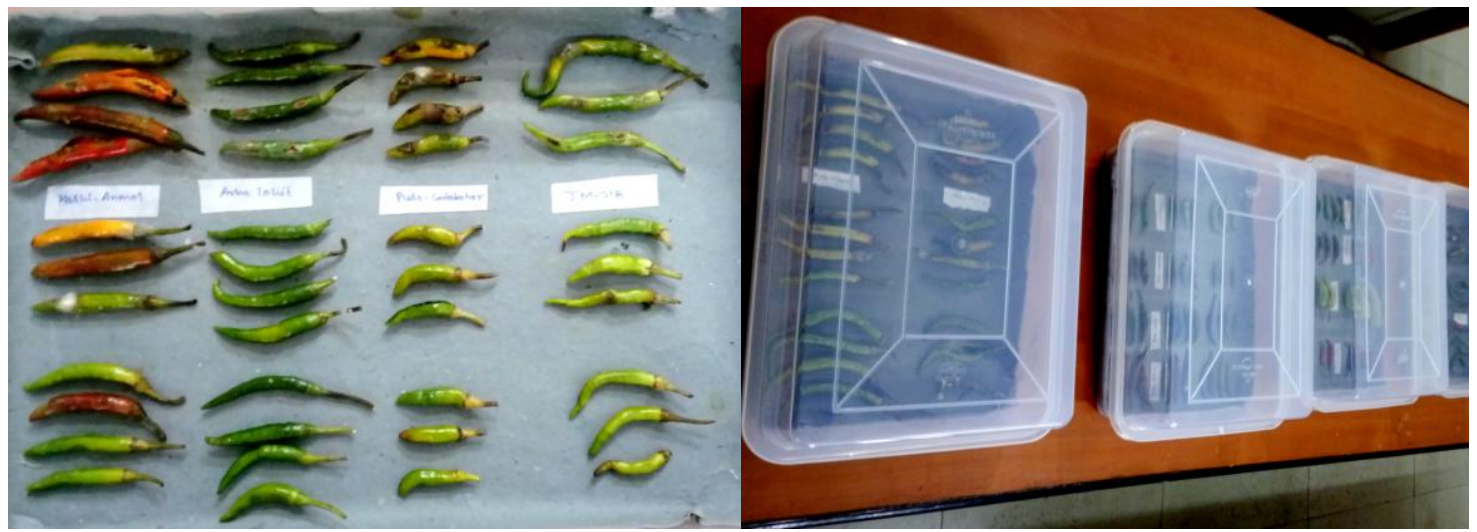

(Treatment - Distilled Water Inoculation, Without injury inoculation, and Injury inoculation)

Technique of artificial inoculation of spore suspension of C.capsici on detached chilli fruits
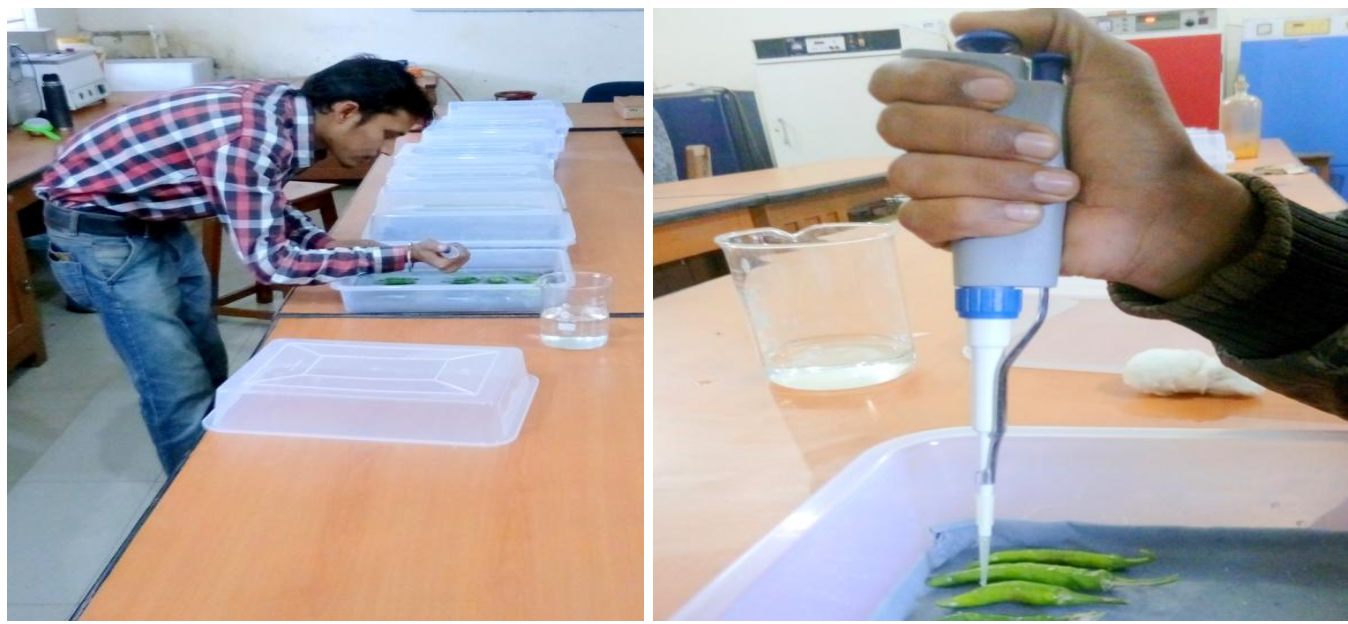


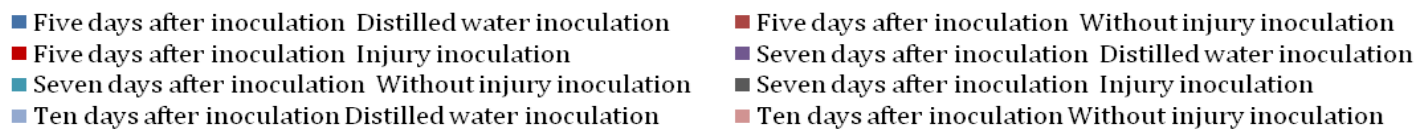

- Ten days after inoculation Distilled water inoculation

- Five days after inoculation Without injury inoculation - Seven days after inoculation Distilled water inoculation - Seven days after inoculation Injury inoculation - Ten days after inoculation Without injury inoculation

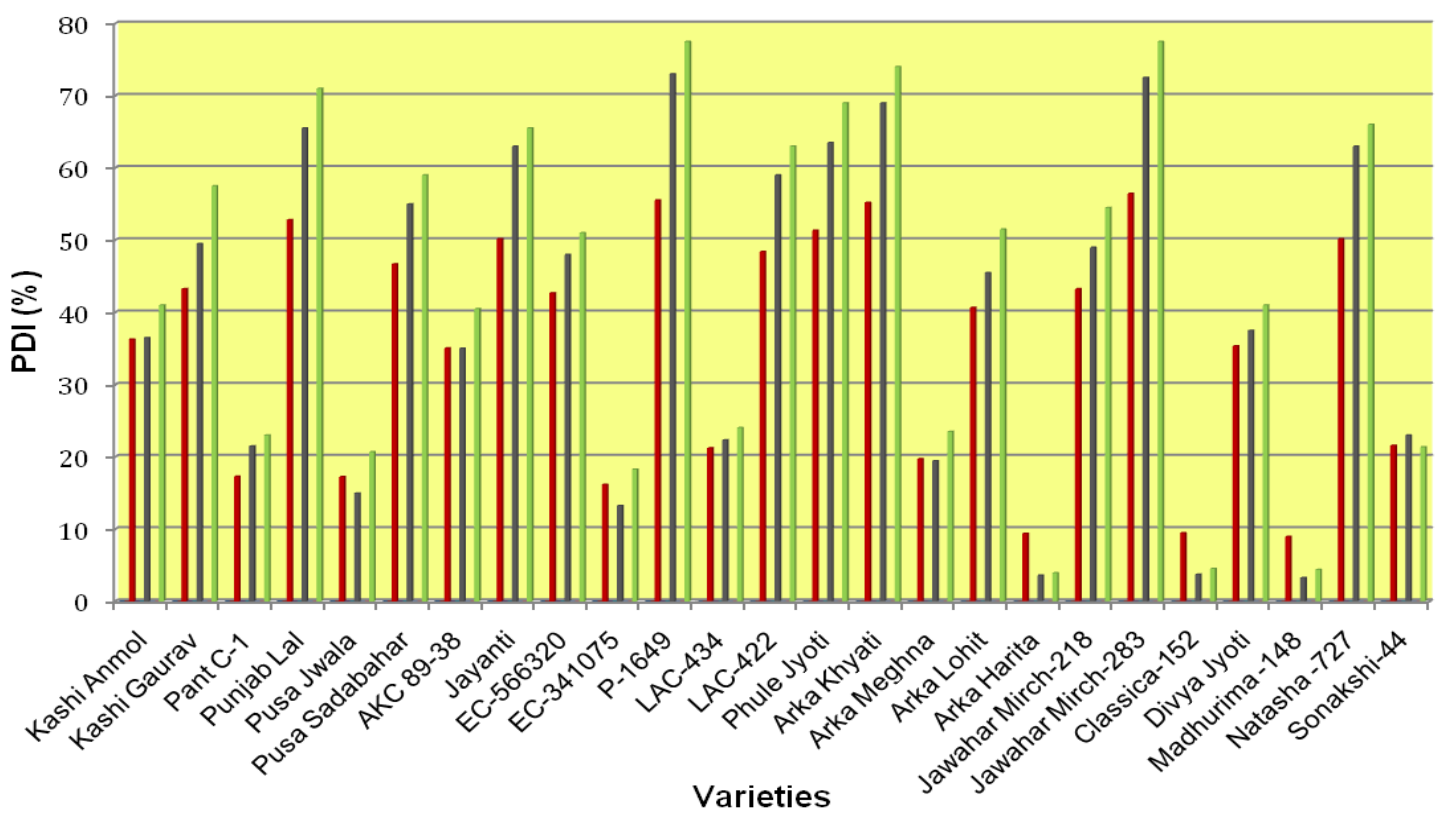

Fig.-: Effect of different methods of inoculation with Colletotrichum capsici after five, seven and ten days of inoculation

It was observed that injury to the fruits favored the $C$. capsici development on detached fruit surface. Varieties Arka Harita, Classica-152 and Madhurima-148 were showing resistance reaction under two drops of conidial suspension after injury the fruits while EC-341075, Pusa Jwala, Pant C-1, Arka Meghna, LAC-434 and Sonakshi-44 were show moderately resistance reaction as compared to rest of varieties at after five, seven and ten days after inoculation. At seven days after inoculation, the data presented in table 1 indicates that all the varieties were show significantly different disease reaction against $C$. capsici after seven days of inoculation. Among the inoculation, two drops of conidial suspension after injury the fruit was notice to be best as compared to other treatments. No infection occurred in without injury inoculated fruits. In varieties, Madhurima-148, Arka Harita and Calssica152 were observed resistant reaction (3.27, 3.61 and $3.75 \%$ respectively) and EC-341075
(13.23\%), Pusa Jwala (14.96\%), Arka Meghna (19.42\%), LAC-434 (22.31\%) Sonakshi-44 (22.99\%) and Pant C-1 (24.50 $\%)$ were showed moderately resistance reaction in two drops of conidial suspension after injury the fruit.

At ten days after inoculation, the data on disease per cent of chilli varieties are presented in table 1. Significant differences occurred with respect to disease per cent in laboratory condition. The data in table 1 shows that disease percentage of all varieties was significantly different. The mean ranged after 10 days inoculation of conidial suspension between 1.31 to $25.83 \%$. Significantly highest disease per cent $(46.05 \%)$ was recorded in two drop of spore suspension after injury the fruit as compared to rest treatments. Varieties Arka Harita, Madhurima-148 and Classica-152 were showing resistance reaction in two drops of spore suspension after injury the fruits (3.93, 
4.39 and $4.51 \%$ respectively) while EC341075, Pusa Jwala, Sonakshi-44, Pant C-1, Arka Meghna and LAC-434 were show moderately resistance reaction $(18.28,20.68$, $21.37,21.75,23.51$ and $24.03 \%$ respectively) as compared to rest of varieties.

Similar results were obtained by Rajamanickam and Sethuraman (2014). They were tested four methods of inoculation under in vitro condition to assess the effectiveness of infection. Among the various methods of inoculation, Spray spore suspension after pinpricking the fruits method was enabled the maximum infection.

Among the three treatment of artificial inoculation, two drops of conidial suspension after injury the fruit was found to be best out of twenty five varieties none of entry was immune reaction. The three entries viz. Arka Harita, Classica-152 and Madhurima-148 showed resistant reaction while six varieties (EC-341075, Pusa Jwala, Pant C-1, LAC-434, Arka Meghna, Divya Jyoti) exhibited moderately resistant at after ten days of fungal inoculation suspension under the laboratory condition.

\section{Acknowledgement}

The authors express thanks to Head, Department of Plant Pathology, and Advisory Committee College of Agriculture, RVSKVV, Gwalior and Head, Krishi Vigyan Kendra RVSKVV Gwalior (M. P.) for providing all the facilities to conduct work.

\section{References}

Anonymous, NHB (2013). Indian Horticulture Database. National Horticultural Board. India: Ministry of Agriculture, Government of India. $\mathrm{p}$. 245.

Bailey, J. A. and Jeger, M. J. (Eds) (1992). Colletotrichum: Biology, Pathology and Control. Wallingford: Commonwealth Myco. Inst., p. 388.

Bansal, R.D., Grover, R.K., 1969.Reaction on chilli (Capsicum fruitenscens) varieties to Colletotrichum capsici. Journal of Research PAU, 6: 345-348.

Jeyalakshmi, C. and Seetharaman, K. (1998). Biological control of fruit rot and die back of chilli with plant products and antagonistic microorganisms. $\mathrm{Pl}$. Dis. Res. 13:46-48.

Rajamanickam, S. and Sethuraman, K. (2014). Effective method of inoculation, virulence, age of susceptibility against Colletotrichum capsici (Syd.) Butler and Bisby, causing anthracnose of chill (Capsicum annuum L.). Trends in Biosci., 7(17): 2500-2503.

Rajapakse R G A S (1998) Observation on the anthracnose of chilli pepper (Capsicum annuum L.) caused by Collectotrichum spp in Sri Lanka. PhD thesis, University of London UK.

Singh, H.P.; Kaur, S. and Singh, J. (1993). Determination of infection in fruit rot (Colletotrichum capsici) of chilli (Capsicum annuum). Indian. J. Agric. Sci. 63: 310-312.

\section{How to cite this article:}

Screening of Different Chilli Genotypes against Anthracnose Disease (Colletotrichum capsici) under Controlled Condition. Int.J.Curr.Microbiol.App.Sci. 7(03): 2328-2334. doi: https://doi.org/10.20546/ijcmas.2018.703.273 